\title{
ABDOMINAL COCOON SYNDROME
}

Minakshi Gadhire, Mansha. B. Singh, Mohan. A. Joshi
1. Associate Professor. Department of General Surgery, Lokmanya Tilak Municipal and General Hospital Mumbai.
2. Senior Registrator. Department of General Surgery, Lokmanya Tilak Municipal and General Hospital Mumbai.
3. Professor, Department of General Surgery, Lokmanya Tilak Municipal and General Hospital Mumbai.

\author{
CORRESPONDING AUTHOR: \\ Mansha. B. Singh, \\ 802, Nisarg building, \\ Off Nargisdutt road, Pali hill, \\ Bandra, Mumbai-50. \\ E-mail: singhmansha@gmail.com
}

ABSTRACT: Abdominal cocoon syndrome is characterized by encapsulation of the small bowel by a fibrous peritoneal sac. We describe the case of a middle aged male patient who presented with sub acute intestinal obstruction. Treatment involves exploratory laparotomy, excision of the sac and adhesiolysis. Early post operative small bowel obstruction is a rare entity in this disease and can be managed conservatively. It is important to have a high degree of suspicion as Abdominal cocoon syndrome has variable clinical presentation and non specific imaging findings.

INTRODUCTION: Sclerosing peritoneal encapsulation is a rare developmental malformation characterized by the encasement of all or part of the small bowel by a thick, accessory peritoneal sheath [1]. It was first described by Owtschinnikow in 1907 as "peritonitis chronica fibrosa incapsulata" [2] and termed "abdominal cocoon" by Foo in 1978 [2,3] .It is characterized by a complete or partial encasement of the small bowel by a thick and fibrotic membrane (cocoons) The loops within the cocoon are normal and adherent to each other. Abdominal cocoon generally presents as recurrent acute or sub acute intestinal obstruction with or without a mass [4]. The ideal treatment is operative, with dissection of the membrane from the intestine and separation of adherent loops of the small bowel until they are laid free and return to their normal configuration. [5]

CASE REPORT: A 35-year-old male presented with complains of multiple episodes of vomiting, constipation, and abdominal discomfort since twenty days. He suffered similar complaints about a month previously for which he underwent a diagnostic laparoscopy at a small nursing home, which revealed cocooning of small bowel loops. Patient was investigated for Tuberculosis of the abdomen; however ascitic fluid ADA levels and ESR were not suggestive of Tuberculosis. Physical examination revealed a mildly tender lump in the Left hypochondrium with minimal abdominal distension. There was no hyperperistalsis on auscultation of the abdomen. Patient was otherwise vitally stable and systemic examination was normal.

Blood parameters were all within normal limits. X-ray erect abdomen revealed 4-5 air fluid levels; USG abdomen showed clumping of small bowel loops in the Left hypochondrium with distal small intestine free. A provisional diagnosis of sub acute intestinal obstruction was made and patient was kept nil per oral, nasogastric decompression and IV hydration was administered. CECT abdomen was done which revealed clumping of jejunal and ileal loops 
surrounded by thickened mesentery and omentum giving a cocoon appearance. Multiple, thin, peritoneal fibrotic strands seen intervening small bowel loops with mild jejunal wall thickening. Patient underwent exploratory laparotomy through midline incision on Day 3 of admission. The entire small bowel was encased in a cocoon-like fibrous membrane. [Fig 1] The loops of small bowel within this membrane were further encased in 3 separate cocoon like sacs with flimsy inter bowel adhesions. The transverse colon and anterior surface of ascending and descending colon as also the visceral surface of the liver [Fig2] and stomach were also covered with similar thin fibrous tissue. The membrane was peeled off the small bowel and extensive adhesiolysis performed by sharp and blunt dissection to free the entire small bowel from DJ flexure to IC junction thus relieving the obstruction [Fig 3]. The fibrous tissue of the sac was sent for histopathological examination, which revealed inflammatory infiltrates. Abdomen was closed by mass closure technique with a drain in pelvis.

Immediate postoperative course was uneventful, patient started on orals on post operative day 5 and discharged on day 8. However on 20th postoperative day the patient presented with multiple episodes of vomiting and colicky pain in abdomen. X-ray abdomen was normal and CT abdomen revealed adherent loops of small bowel to anterior abdominal wall; however there was free passage of oral contrast up to the descending colon. Patient was managed conservatively with nasogastric decompression and IV fluid rehydration and discharged on day 10.3 months post surgery he remains in good health.

DISCUSSION: Abdominal cocoon may be classified into primary or idiopathic and secondary forms [7]. Primary abdominal cocoon occurs mainly in young women from tropical and subtropical zones. Although retrograde menstruation with or without viral infection of the fallopian tubes has been suggested as a possible etiology [7,8], it does not account for the occasional occurrence of abdominal cocoon in males [2]. Secondary abdominal cocoon is apparently associated with predisposing factors, such as recurrent peritonitis, intake of intraperitoneal irritants, including antibiotics and beta blockers, chronic ambulatory peritoneal dialysis (CAPD), sarcoidosis, familial Mediterranean fever, carcinoid syndrome, exposure to asbestos, and autoimmune disease [7,9]. The clinical presentation of abdominal cocoon includes acute, sub acute, or chronic intestinal obstruction, abdominal distension, nausea, and vomiting $[2,9]$. Patients usually complain of recurrent attacks of intestinal obstruction. An accurate diagnosis is difficult to make preoperatively [5] because findings on biochemical investigations are usually normal, and imaging findings are nonspecific [7], although plain abdominal X-ray film may show air- fluid levels [8]. In the rare reports of the CT appearance of abdominal cocoon, adherent small bowel loops encased within a thick enhancing peritoneal membrane were visualized [2,7].Other CT features of abdominal cocoon include signs of obstruction, agglutination and the fixation of intestinal loops, mural thickening, ascites and localized fluid collections, peritoneal thickening and enhancement, peritoneal or mural calcifications and reactive adenopathy [10].

The typical finding at surgery is a conglomeration of small bowel loops encased in a dense white membrane [1,7]. Involvement of stomach and visceral surface of liver as in this case is extremely rare. Treatment, as in the present case, consists of excision of the accessory peritoneal sac with lysis of the interloop adhesions. Bowel resection is unnecessary $[1,5]$ unless a nonviable segment is found [7].

Abdominal cocoon presenting with early post operative small bowel obstruction is extremely rare entity. 
Most authors have applied a definition of EPSBO similar to that of Ellozy et al, who diagnosed EPSBO when crampy abdominal pain, vomiting, and radio- graphic findings consistent with intestinal obstruction were present after an initial return of bowel function within 30 days after surgery $[11,12]$. The majority of episodes of EPSBO are most probably caused by adhesions.

Most instances of mechanical EPSBO can be treated expectantly for at least 10 to 14 days with almost no risk of bowel strangulation. Many episodes of EPSBO resolve spontaneously with no cause identified [12].

It is noteworthy that EPSBO is a postoperative complication associated with surgery for abdominal cocoon after extensive adhesiolysis. Many factors con- tribute to the development of EPSBO after surgery for abdominal cocoon. First, both abdominal cocoon and EPSBO are associated with enhanced collagen production and subsequent fibrosis. [12, 13]. Second, efforts to divide adhesions may further precipitate and increase their formation. Prevention includes sound operative techniques, attention to detail, gentle dissection and handling of tissues, careful hemostasis to avoid hematoma formation, and thorough peritoneal cleansing, leaving as little foreign material as possible (for example, non-absorbable suture material). [12]

CONCLUSION: Abdominal cocoon syndrome is a rare cause of small bowel obstruction. The non-specific clinical picture and imaging findings make it difficult to diagnose. In Indian settings it is most important to rule out Tuberculosis in such patients .A high index of suspicion is required and CT done pre operatively may help in confirmation of diagnosis. Treatment involves excision of the fibrous sac with bowel adhesiolysis taking care to keep dissection to a minimum with gentle handling of bowel. Outcome is generally good.

\section{REFERENCES:}

1. Mordehai J, Kleiner O, Kirshtein B, Barki Y, Mares AJ. Peritoneal encapsulation: a rare cause of bowel obstruction in children. J Pediatr Surg 2001; 36(7):1059-1061.

2. Lalloo S, Krishna D, Maharajh J. Case report: abdominal cocoon associated with tuberculous pelvic inflammatory disease. Br J Radiol 2002; 75(890):174-176.

3. Tsunoda T, Mochinaga N, Eto T, Furui J, Tomioka T, Takahara H. Sclerosing encapsulating peritonitis combined with peritoneal encapsulation. Arch Surg 1993;128:353-5

4. Foo KT, Ng KC, Rauff A, et al. Unusual small intestinal obstruction in adolescent girls: the ab- dominal cocoon. Br J Surg. 1978;65:427-430

5. Yoon YW, Chung JP, Park HJ, Cho HG, Chon CY, Park IS, et al. A case of abdominal cocoon. J Korean Med Sci 1995; 10(3):220-225.

6. Kumar M, Deb M, Parshad R. Abdominal Cocoon: Report of a Case. Surg Today. 2000;30:950-953.

7. Al-Abassi AA, Emad M. Abdominal cocoon. An unusual cause of intestinal obstruction. Saudi Med J 2004; 25(10):1482-1485.

8. Ahmed MN, Kaur S, Zargar HU. Abdominal cocoon: an unusual intestinal obstruction. J Postgrad Med 1984; 30(1):62-63.

9. Dequanter D, Lefebvre JC, De Pauw L, Nortier J, Kinnaert P. Sclerosing peritonitis: report of three cases. ActaChirBelg 2003; 103(4):408-411.

10. Krestin GP, Kaci G, Hauser M et al. Imaging diagnosis of sclerosing peritonitis and radiological signs to the extent of the disease. Abdom Imaging 1995;20:414-420

11. Ellozy SH, Harris MT, Bauer JJ, et al. Early post- operative small-bowel obstruction: a prospective evaluation in 242 consecutive abdominal opera- tions. Dis Colon Rectum. 


\section{2; $45: 1214-1217$}

12. Sajja SB, Schein M. Early postoperative small bowel obstruction. Br J Surg. 2004;91:683-691.

13. Lin $\mathrm{CH}, \mathrm{Yu}$ JC,Chen $\mathrm{TW}$, et al. Sclerosing encapsulating peritonitis in a liver transplant patient: a case report. World J Gastroenterol. 2005;11:5412- 5413.

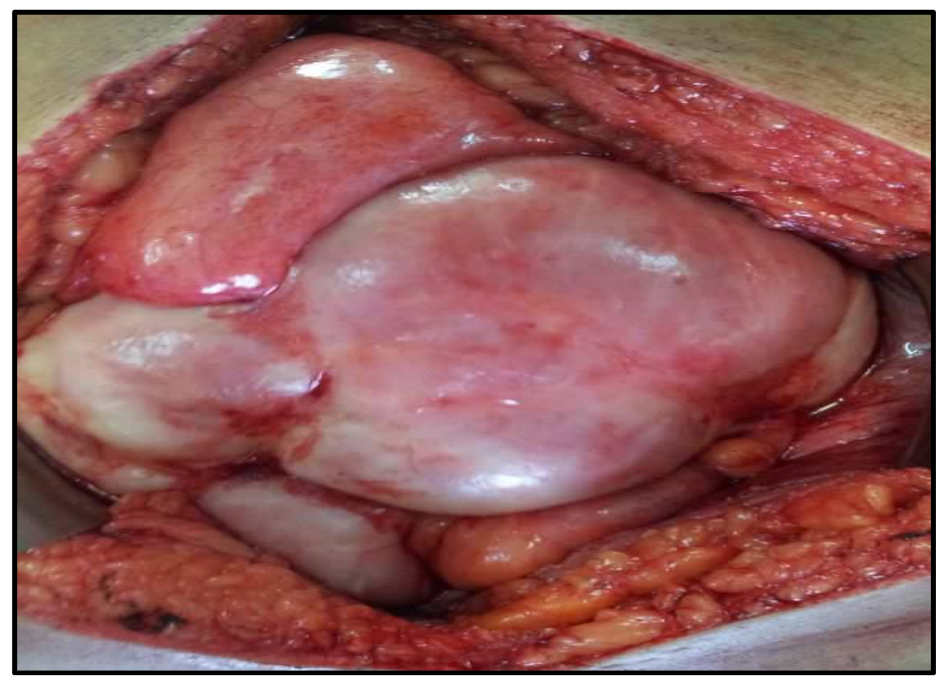

FIG 1: Small bowel encased in fibrous peritoneal sac

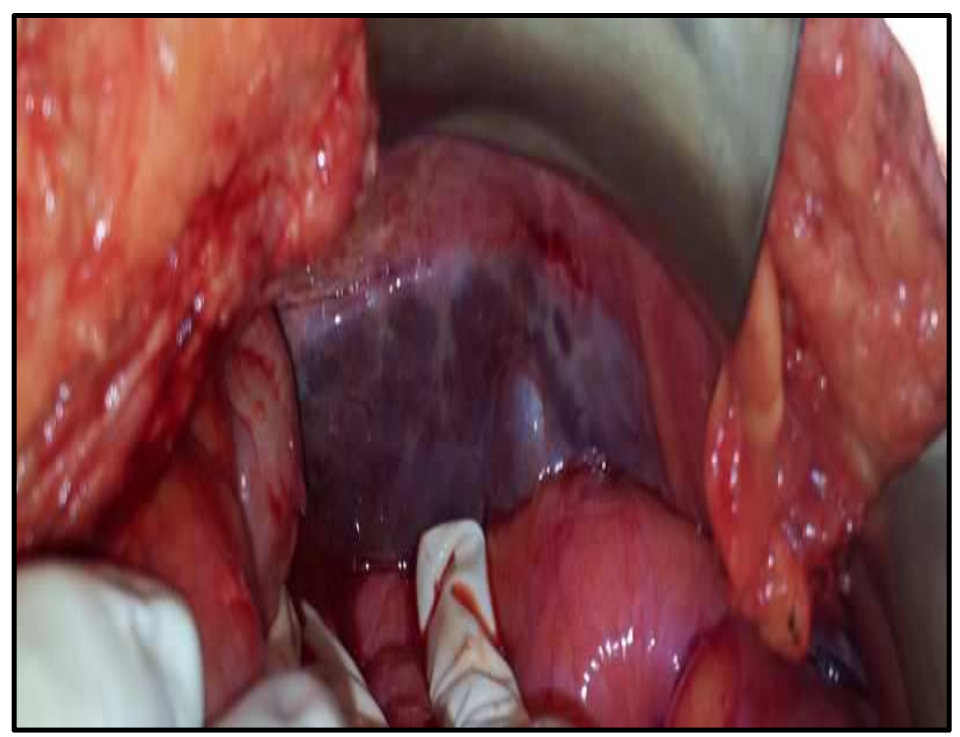

FIG 2: Visceral surface of liver covered with fibrous layer 


\section{CASE REPORT}

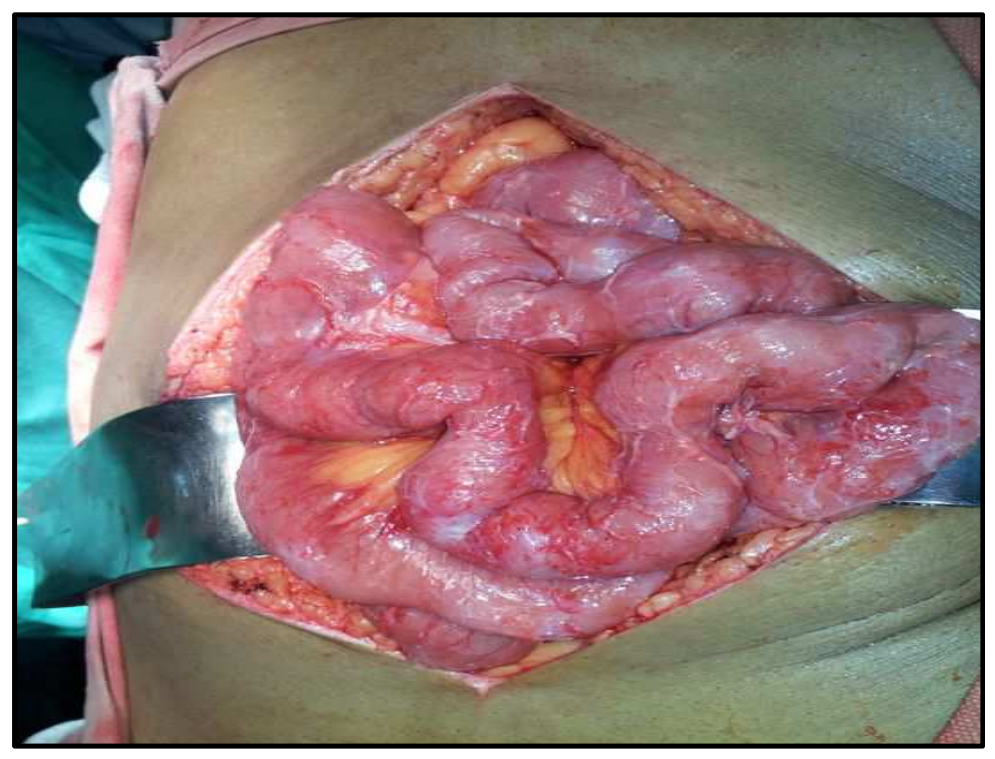

FIG 3: Separated loops of small bowel after excision of sac and adhesiolysis 